\title{
Versão brasileira do Preschool-age Children's Physical Activity Questionnaire (Pre-PAQ)
}

\author{
Brazilian version of the Preschool-age Children's Physical Activity Questionnaire (Pre-PAQ) \\ Versión brasileña del Preschool-age Children's Physical Activity Questionnaire (Pre-PAQ) \\ Nathalia Trasmonte Silva', Cristina dos Santos Cardoso de Sá2, Mariana Callil Voos³, \\ Raquel de Paula Carvalho ${ }^{4}$
}

\begin{abstract}
RESUMO | Foi realizada a tradução e a adaptação transcultural do Preschool-age Children's Physical Activity Questionnaire para a língua portuguesa do Brasil. O questionário foi traduzido em duas versões diferentes por dois profissionais proficientes na língua inglesa. Baseada nessas duas traduções independentes, foi criada a tradução única, então retrotraduzida para o inglês por outros dois tradutores. As retrotraduções passaram por novo processo de consenso, que gerou uma versão única, que foi comparada com o questionário em inglês original. A versão do questionário em português do Brasil, denominada Questionário de Atividade Física de Crianças em Idade Pré-Escolar (Pre-PAQ), foi revisada por uma comissão de especialistas para verificação do conteúdo e gerou a segunda versão de concordância. Em seguida, 28 profissionais da área avaliaram o questionário quanto à adequação para a língua portuguesa do Brasil e à adaptação cultural para crianças brasileiras de 3 a 5 anos de idade. Conclui-se que as etapas de tradução e adaptação cultural foram concluídas com sucesso, ainda que futuros estudos para testar a confiabilidade e validade do conteúdo da versão brasileira do Pre-PAQ sejam necessários para garantir a acurácia da aplicação na população-alvo.
\end{abstract}

Descritores I Tradução; Cultura; Atividade Motora; Criança.

ABSTRACT | The translation and cross-cultural adaptation of the Preschool-age Children's Physical Activity Questionnaire into Brazilian Portuguese was carried out. The questionnaire was translated into two different versions by two professionals proficient in the English language. Based on these two independent translations, a single translation was created, which was then back translated into English by two other translators. The back-translations went through a new consensus process, which generated a single version, that was compared with the original English questionnaire. The Brazilian Portuguese version of the questionnaire, called "Questionário de Atividade Física de Crianças em Idade Pré-Escolar (Pre-PAQ)", was reviewed by a committee of experts to verify the content and generated the second version of accordance. Then, 28 professionals in the field evaluated the questionnaire regarding the suitability for the Brazilian Portuguese language and the cultural adaptation for Brazilian children from 3 to 5 years old. It is concluded that the stages of translation and cultural adaptation were successfully concluded, although future studies to test the content reliability and validity of the Pre-PAQ's Brazilian version are necessary to ensure the application accuracy in the target population.

Keywords I Translation; Culture; Motor Activity; Child.

RESUMEN I Se ha realizado una traducción y adaptación transcultural del Preschool-age Children's Physical Activity Questionnaire al idioma portugués de Brasil. El cuestionario fue traducido por dos profesionales con dominio del inglés para dos versiones diferentes. Sobre la base de estas dos traducciones independientes, se creó una única traducción, que luego se volvió a traducir al inglés por otros dos traductores. Las retrotraducciones pasaron por un nuevo proceso de consenso, lo cual generó una única versión para comparar con el cuestionario original en inglés. La versión del cuestionario al portugués brasileño, llamada Questionário de Atividade Física de Crianças em Idade

IUniversidade Federal de São Paulo - Santos (SP), Brasil. E-mail: nathalia.trasmonte@gmail.com. Orcid: 0000-0003-4598-0842 2Universidade Federal de São Paulo (Unifesp) - Santos (SP), Brasil. E-mail: cristina.sa@uol.com.br. Orcid: 0000-0002-0920-6668 ${ }_{3}^{3}$ Pontifícia Universidade Católica - São Paulo (SP), Brasil. E-mail: mcvoos@pucsp.br. Orcid: 0000-0001-6252-7287

${ }^{4}$ Universidade Federal de São Paulo (Unifesp) - Santos (SP), Brasil. E-mail: raquelpcarvalho@gmail.com. Orcid:0000-0002-5261-7235 
Pré-Escolar (Pre-PAQ), fue revisada por un comité de expertos para verificar el contenido y generó la segunda versión del acuerdo. Luego, 28 profesionales en el campo evaluaron el cuestionario en cuanto a la idoneidad para el portugués brasileño y la adaptación cultural para niños brasileños de 3 a 5 años. Se concluye que las etapas de traducción y adaptación cultural fueron exitosas, aunque son necesarios futuros estudios para probar la confiabilidad y validez de contenido a la versión brasileña del Pre-PAQ para asegurar la precisión de la aplicación en la población objetivo. Palabras clave | Traducción; Cultura; Actividad Motora; Niño.

\section{INTRODUÇÃO}

A fase pré-escolar é importante na vida da criança para estabelecer rotinas e comportamentos promotores de saúde. No Brasil, essa fase equivale de 4 a 6 anos de idade, sendo a média de permanência diária das crianças na escola de nove horas ${ }^{1}$. Atualmente, as crianças têm, cada vez mais, apresentado peso acima do esperado, pouca prática de atividade física $(\mathrm{AF})$ e excesso de uso de telas, como tablets e celulares ${ }^{2}$. Os principais fatores que determinam o perfil de comportamento sedentário em pré-escolares são os hábitos de $\mathrm{AF}$ dos pais ${ }^{3}$ e o tempo de exposição excessivo a telas ${ }^{4}$.

$\mathrm{O}$ ambiente é um fator que influencia positivamente a prática de AF nessa faixa etária. Locais como parques do bairro ou playground de condomínio motivam os pais ou cuidadores a levarem as crianças para vivenciar brincadeiras fisicamente ativas ${ }^{5}$. Além disso, ambientes como creches e escolas também exercem esse papel ${ }^{5}$.

Dada a importância da prática de AF na infância, independentemente da idade, é recomendável saber como mensurá-la ${ }^{6}$ para avaliar a inclinação do sujeito a praticar AF, averiguar a relação entre a $\mathrm{AF}$ e a saúde e examinar a eficácia de intervenções. Hidding et al. ${ }^{6}$ sugerem o uso de acelerômetros, que fornecem medidas válidas e confiáveis, porém não concedem informações das características e circunstâncias de comportamento. Como alternativa, sugere-se o uso de questionário de autorrelato, considerados adequados e baratos para avaliar a prática de $\mathrm{AF}$ e oferecem informações sobre o ambiente e o contexto em que a pessoa está inserida ${ }^{7}$. $\mathrm{Na}$ idade pré-escolar, as crianças ainda não apresentam capacidade cognitiva para avaliar, qualificar ou recordar as atividades que praticaram ${ }^{7}$, sendo os questionários baseados nas respostas dos pais a medida preferida para avaliar a $\mathrm{AF}$ nessa faixa etária ${ }^{8}$.

O Preschool-age Children's Physical Activity Questionnaire (Pre-PAQ) é um questionário elaborado na Austrália com a finalidade de mensurar os níveis de prática de AF e de sedentarismo de pré-escolares no ambiente doméstico, sendo as perguntas respondidas pelos pais ${ }^{9}$.
O Pre-PAQ foi elaborado a partir do pressuposto de que existem fatores multidimensionais que são reflexos das estruturas socioecológicas a que as crianças estão inseridas, influenciando o seu comportamento. Em relação às propriedades psicométricas, o Pre-PAQ apresenta tanto confiabilidade quanto validade adequada, variando de moderada a muito boa ${ }^{7}$. O questionário é composto por itens que abordam especificamente: hábitos e atitudes relacionados à prática de $\mathrm{AF}$ praticada pelos pais da criança que está sendo avaliada; dados demográficos da família; ambiente familiar e vizinhança e atividades inerentes à infância ${ }^{7}$ - como correr, pular, andar de bicicleta, assistir televisão, entre outras.

Para avaliar quais atividades físicas são realizadas pelas crianças, o Pre-PAQ apresenta uma lista de atividades típicas da idade pré-escolar em que as opções de respostas são “sim” ou “não". Se a resposta for "sim”, é perguntado o tempo que a criança gastou realizando a atividade. Para avaliar os hábitos dos pais, eles informam quais tipos, duração e número de vezes que realizam determinada AF por semana ${ }^{7}$.

Muitas das avaliações usadas na pesquisa e prática clínica são desenvolvidas e validadas em países de língua inglesa; dessa forma, existe a necessidade de pesquisadores nativos de outros idiomas traduzi-las e validá-las para o contexto cultural de seu país ${ }^{10}$. O processo de adaptação cultural compreende a análise dos termos linguísticos utilizados para a aplicação do questionário em um ambiente diferente daquele para o qual ele foi elaborado ${ }^{11}$.

No Brasil, há o interesse na tradução de testes já padronizados em idiomas estrangeiros em vez da criação de novos testes na língua portuguesa ${ }^{12}$. A tradução e a adaptação cultural são recomendadas pela Organização Mundial da Saúde (OMS) por favorecerem a comparação dos resultados de pesquisas realizadas em diferentes países e a comunicação entre os pesquisadores ${ }^{13}$.

Os questionários que investigam os níveis de $\mathrm{AF}$ traduzidos para o português do Brasil limitam-se à faixa etária superior da abordada no Pre-PAQ ${ }^{14}$, como, por exemplo, os questionários PAQ-C (que avalia crianças 
com idade entre 8 e 13 anos) e PAQ-A (para adolescentes entre 14 e 18 anos). Na revisão bibliográfica feita para este estudo, não foram encontrados questionários em português para crianças em idade pré-escolar, reforçando a necessidade de tradução de um questionário para essa população. Com isso, este estudo objetivou a tradução e a adaptação cultural do Preschool-age Children's Physical Activity Questionnaire para a língua portuguesa do Brasil.

\section{MÉTODO}

Foi solicitada, incialmente, a autorização da autora do Pre-PAQ original por meio do envio da documentação descrevendo todas as etapas para a tradução e a adaptação cultural. O segundo passo foi a tradução para a língua portuguesa do Brasil de acordo com as recomendações internacionais ${ }^{11}$; essa etapa foi realizada por dois tradutores independentes, somente um deles tendo ciência dos objetivos do estudo ( apesar de não conhecer o questionário). As duas traduções para o português do Brasil (denominadas, respectivamente, T1 e T2) foram fundidas em uma versão traduzida única (TU) depois do consenso entre os tradutores e pesquisadores.

A seguir, a TU foi retrotraduzida (RT) para o idioma inicial (inglês), seguindo as mesmas regras da tradução inicial, que desconheciam as finalidades do estudo. Essas duas versões em inglês (RT1 e RT2) passaram por novo processo de consenso entre tradutores e pesquisadores, resultando em uma versão retrotraduzida final (RTfinal) em inglês, que foi comparada com a versão original com a finalidade de identificar possíveis diferenças semânticas.

A versão do questionário em português do Brasil (TU) foi revisada pela comissão de especialistas composta por dois fisioterapeutas e um terapeuta ocupacional, todos com experiência de pelo menos cinco anos na área da infância e com conhecimento dos dois idiomas, para verificação da validade do conteúdo. A comissão comparou a versão em português do Brasil,item por item, com a versão original em inglês para confrontar sua concordância e sugerir mudanças que poderiam aprimorar a tradução. Cada item também foi avaliado quanto à relevância na avaliação do conteúdo do instrumento, verificando a equivalência. Após essa revisão, a versão retrotraduzida final (RTfinal) foi enviada a autora do questionário original e, com a aprovação da versão RTfinal, obteve-se à versão traduzida final (Tfinal) em português do Brasil. A Figura 1 apresenta o fluxograma que esquematiza o processo de tradução até a obtenção da versão final (Tfinal).

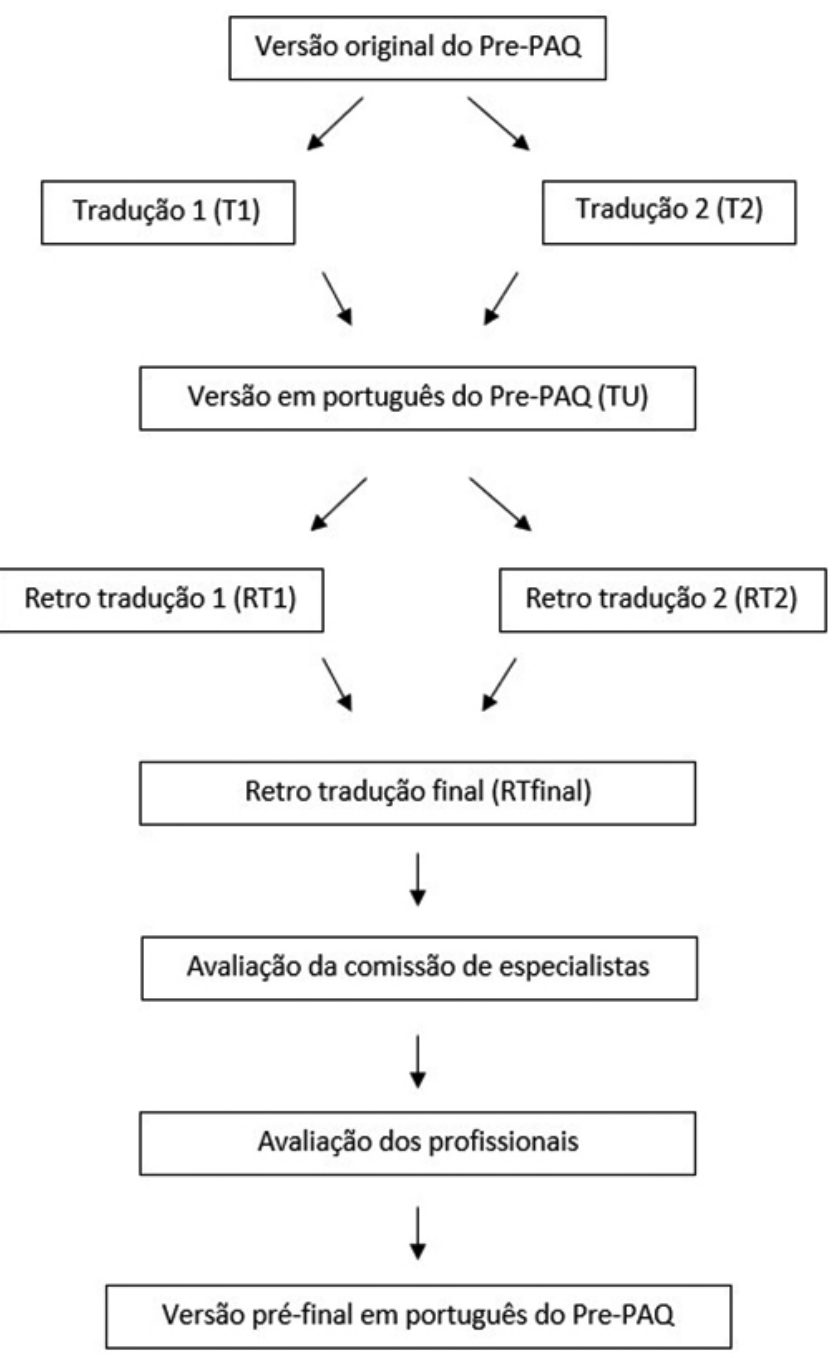

Figura 1. Fluxograma do processo de tradução

A Tfinal foi entregue a 28 profissionais com no mínimo dois anos de experiência na área da fisioterapia, terapia ocupacional ou educação física para que adaptações culturais pudessem ser incorporadas ao questionário sem que isso resultasse em alterações de conteúdo. Esses profissionais avaliaram o questionário item a item e preencheram uma tabela de checagem, na qual havia espaço para descreverem sugestões aos itens que julgassem não condizer com a realidade da criança brasileira na faixa etária entre 4 e 6 anos de idade. Poderiam, ainda, ser apontadas sugestões a respeito da adequação à língua portuguesa.

Cada profissional avaliou o questionário Pre-PAQ em relação à descrição das instruções e formulário para avaliação, classificando-os em: adequado como está; necessidade de inclusão de alguma questão/informação; necessidade de exclusão de alguma questão informação ou necessidade de modificação de alguma questão/ informação. A partir das informações recebidas, foi 
organizado um banco de dados para sistematizar a revisão da Tfinal, tomando-se o devido cuidado que o conteúdo do questionário original em inglês não fosse modificado. Após essa revisão, obteve-se a versão brasileira do Pre-PAQ.

\section{RESULTADOS}

Feita a tradução, a avaliação foi denominada versão brasileira do "Questionário de Atividade Física de Crianças com Idade Pré-Escolar (Pre-PAQ)". Especialistas revisaram e compararam a TU com a versão original em inglês e foram destacados os tópicos que causaram dificuldade na compreensão e na interpretação das questões. Assim, estes tópicos foram modificados (Quadros 1 e 2).

Com relação à descrição das instruções de aplicação da versão em português do Pre-PAQ todos os profissionais relataram que estavam adequadas, salvo duas sugestões feitas pelos especialistas (Quadro 3).

Quadro 1. Alterações no instrumento após análise dos especialistas

\begin{tabular}{|c|c|c|}
\hline Seção & Descrição do item & Adequações realizadas \\
\hline \multirow[t]{2}{*}{1} & \multirow[t]{2}{*}{ Informações gerais } & $\begin{array}{l}\text { Adicionado na orientação de aplicação do questionário: } \\
\text { "Se seu filho passa o dia inteiro em cuidados formais, peça ajuda ao principal cuidador fora de } \\
\text { casa (ex.: professor)" }\end{array}$ \\
\hline & & Trocar "nunca se casou" por "solteiro" \\
\hline \multirow{3}{*}{2} & \multirow{3}{*}{ Atividades físicas e hábitos dos pais } & $\begin{array}{l}\text { Dada a dificuldade de quantificar o tempo de prática de atividade física, foi adicionado na } \\
\text { orientação de aplicação que esta medida é a "estimativa do tempo" e não o "tempo exato" de } \\
\text { prática }\end{array}$ \\
\hline & & Retirada a medida "1/1/4 de acre", que não utilizamos no Brasil \\
\hline & & Substituído o DVD pelo uso do celular \\
\hline 3 & Casa e vizinhança & Substituído o DVD pelo uso do celular \\
\hline 4 & Sua criança & Verificar linguagem acessível à uma pessoa leiga, por exemplo: marchou; trotou \\
\hline
\end{tabular}

Quadro 2. Alterações no instrumento após análise dos especialistas

\begin{tabular}{|c|c|c|}
\hline Item & Descrição do item & Adequações realizadas \\
\hline Q4 & Qual seu nível de escolaridade mais alto? & $\begin{array}{l}\text { Sugestão: Fundamental completo / fundamental incompleto / ensino médio completo / } \\
\text { ensino médio incompleto / curso técnico ou profissionalizante completo / curso técnico } \\
\text { ou profissionalizante incompleto / superior completo / superior incompleto }\end{array}$ \\
\hline \multirow{2}{*}{ Q6 } & \multirow{2}{*}{ Qual é o idioma falado em casa? } & Retirar: grego, cantones, árabe, vietnamita e mandarim \\
\hline & & Incluir: português, espanhol, inglês, francês, japonês, chinês, italiano e outros \\
\hline \multirow{4}{*}{ Q11a } & \multirow{4}{*}{$\begin{array}{l}\text { Na semana passada, quantas vezes você fez } \\
\text { qualquer outra atividade física moderada que } \\
\text { não tenha sido mencionada (ex. Natação suave, } \\
\text { tênis socialmente, golfe etc.) }\end{array}$} & Adicionado: pilates \\
\hline & & Adicionada: natação \\
\hline & & Adicionados: corrida, futebol, academia e pilates \\
\hline & & Retirada: tênis \\
\hline \multirow[b]{2}{*}{ Q12a } & \multirow{2}{*}{$\begin{array}{l}\text { Na semana passada, quantas vezes você } \\
\text { praticou atividade física intensa que fez } \\
\text { com que você precisasse respirar com maior } \\
\text { frequência e profundamente (ex. corrida, } \\
\text { ciclismo, tênis competitivo, jardinagem ou } \\
\text { trabalho braçal pesado etc) }\end{array}$} & Retirada: jardinagem \\
\hline & & Adicionado: futebol \\
\hline \multirow{5}{*}{ Q13 } & \multirow{5}{*}{$\begin{array}{l}\text { Quanto você estimaria que foi o tempo total } \\
\text { que você gastou assistindo TV, vídeos, ou DVDs } \\
\text { como sua principal atividade na SEMANA } \\
\text { PASSADA Por favor não inclua o tempo em que } \\
\text { a TV estava ligada, mas você estava fazendo } \\
\text { outra coisa como por exemplo preparando } \\
\text { alguma refeição. }\end{array}$} & Adicionado: celular \\
\hline & & Adicionado: computador - redes sociais e outras atividades \\
\hline & & Adicionado: redes sociais no celular \\
\hline & & Após o termo "por favor" foi adicionada vírgula \\
\hline & & Retirado: DVD \\
\hline
\end{tabular}




\section{Quadro 2. Continuação}

Item Descrição do item

Quanto você estimaria que foi o tempo total que você gastou jogando jogos eletrônicos na

Q14 SEMANA PASSADA. Por favor circule os jogos eletrônicos usados

PlayStation, Nintendo, Xbox, Wii II

Quanto você estimaria que foi o tempo total

Q15 que você gastou usando o computador em casa no seu tempo livre na SEMANA PASSADA. (NÃO inclua horas de uso para trabalhar)

Quanto você concorda com as frases a seguir?

Q16 "Eu sou fisicamente ativo com ou em frente da minha criança"

Q17 O que melhor descreve seu quintal?

Você tem acesso a qualquer uma das

Q18 facilidades abaixo, no seu quintal ou ambiente doméstico?

Quantos de cada objeto baixo que você tem em casa?

Seu bairro tem esses locais ou facilidades onde

Q21 sua criança pode brincar e ser fisicamente ativo? total, SEMANA PASSADA (dias da semana + sábado + domingo)

Quais cuidados ou serviços sua criança frequentou semana PASSADA?

Sua criança come suas refeições em frente da televisão?

Com que frequência sua criança usa as

Q31 facilidades listadas abaixo para brincar e ser fisicamente ativo, em um mês típico quando o clima está favorável?

Quais das seguintes atividades sua criança fez ONTEM?

Como estava o clima no FIANAL DE SEMANA PASSADO?

Quais das seguintes atividades sua criança fez no final de semana passado?

\section{Adequações realizadas}

Adicionado: tablet e smartphones

Adicionado: celular para jogar

Adicionado: celular

Adicionado: computador - redes sociais e outras atividades

Adicionado: celular para jogar

Frase substituída para: "Sou fisicamente ativo em frente à minha criança ou junto com ela"

Especificado que o espaço externo em condomínio é considerado como "quintal não privativo"

Retirado: spa e parede de escalada

Adicionados os sinônimos para SPA: jacuzzi e ofurô

Adicionado: celular

Adicionado: celular que a criança tem para brincar

Adicionadas: brincadeiras na rua ou em vias públicas

Retirado: playground; adicionado: parquinho

Adicionada a opção de tempo gasto em ônibus/transporte público

Adicionado: mototáxi, táxi, uber, transporte escolar

Adicionada na especificação que estão nos cuidados informais: ter com compromisso de ficar com os avós, amigos ou babá

Adicionada a especificação dos cuidados ocasionais: ficar com os avós, vizinhos ou conhecidos por curto período, em ocasiões pontuais como, por exemplo, quando os pais precisam ir ao médico

- Adicionado: tablet e celular

A palavra "todos" foi corrigida para "todas"

Adicionadas: ruas, vias públicas

- Adicionado: uso de celular

- Adicionado: uso de tablet

Adicionado: muito seco para brincar fora de casa

Adicionado o uso de aparelho celular

Adicionado o uso de tablet

Adicionado o uso de patins

Quadro 3. Alterações nas instruções de aplicação após sugestões dos profissionais

\begin{tabular}{c|l|lc} 
Seção & \multicolumn{1}{|c|}{ Sugestão dos profissionais } & \multicolumn{1}{c}{ Adequações realizadas nas instruções de aplicação } \\
\hline $\begin{array}{c}\text { Instruções de } \\
\text { aplicação }\end{array}$ & $\begin{array}{l}\text { A mãe ou o pai pode não passar a } \\
\text { maior parte do dia com a criança, seria } \\
\text { interessante incluir o professor }\end{array}$ & $\begin{array}{l}\text { "[ificuldade do voluntário em recordar } \\
\text { de casa (por exemplo o professor)." } \\
\text { o tempo exato de prática de atividade } \\
\text { física }\end{array}$ & $\begin{array}{l}\text { "[...] Ainda, nas questões desta seção em que é necessário recordar o tempo de } \\
\text { prática de algumas atividades, vale enfatizar para o entrevistado que o questionário } \\
\text { demanda tempo estimado, não é necessário saber ou recordar o tempo exato de } \\
\text { prática." }\end{array}$ \\
\hline
\end{tabular}




\section{DISCUSSÃO}

Este estudo traduziu e adaptou para o português do Brasil o Questionário de Atividade Física de Criança em Idade Pré-escolar (Pre-PAQ), que tem como o objetivo mensurar o nível de prática de AF de crianças entre $3 \mathrm{e}$ 5 anos de idade e descrever os fatores que influenciam na prática.

Originalmente, o Pre-PAQ foi elaborado na língua inglesa voltado para o contexto cultural da criança australiana. Se fez necessário, portanto, fazer adaptação cultural do instrumento avaliativo, dado que, descrito tal como no original, não seria sensível para a coleta dos dados de crianças inseridas na cultura brasileira. Com relação ao contexto gramatical, algumas palavras podiam não apresentar os mesmos significados quando traduzidas no literal; dessa forma, tradução e adaptação cultural garantem que tanto a aplicação quanto a interpretação dos resultados sejam válidas e confiáveis ${ }^{15}$.

No decorrer das fases previstas da tradução, não foram levantadas divergências importantes entre o que foi apresentado pelos tradutores independentes e a comissão de especialistas; As discordâncias, porém, apareceram na etapa da adaptação cultural, em que os profissionais experientes que avaliaram o questionário levantaram uma quantidade importante de atividades que não são praticadas no Brasil e, dessa forma, poderiam prejudicar a acurácia das respostas dadas pelos voluntários em estudos futuros. Por exemplo: prática de golfe, escalada e jardinagem que não são práticas comuns em nossa cultura, independentemente da faixa etária. Essas opções foram substituídas por atividades comuns da prática do brasileiro após aprovação da autora do questionário original em inglês.

Outro fator importante e que merece destaque está relacionado ao avanço tecnológico e como ele modifica os hábitos de uso de dispositivos eletrônicos. O Pre-PAQ original foi criado e publicado na Austrália em 2011, quando o uso de aparelho de DVD, computadores e jogos de videogame era comum em diversas faixas etárias. Agora, passados 10 anos da publicação original em inglês, os profissionais que avaliaram a versão traduzida levantaram a possível tendência, na faixa etária que será alvo do questionário, de substituição desses dispositivos pelo celular ou tablet, que contam, atualmente, com a função smart e sistema operacional que permitem que o usuário faça download de aplicativos de vídeos (substituindo o uso do aparelho do DVD), e de jogos (substituindo o uso do videogame). Apesar de retirados da redação, eles continuam incorporados ao conteúdo nos termos jogos e vídeo, respectivamente. Ainda, para a faixa etária dos pais e cuidadores, as redes sociais passaram a apresentar maior relevância recentemente, aumentando o tempo em que esses usuários passam na frente de telas ${ }^{16}-$ foi necessário, assim, acrescentar esses dispositivos aos itens do questionário.

A próxima etapa foi a realização da tradução, adaptação e validação de conteúdo do Pré-PAQ.Futuros estudos devem ser feitos para confirmar as propriedades psicométricas relacionadas à confiabilidade e à validade de construct para a administração do questionário no público-alvo. Feito isso, o processo integral de tradução, adaptação e validação da versão brasileira do questionário estará concluído e garantirá a acurácia de sua aplicação na população de crianças de 4 a 6 anos de idade.

\section{REFERÊNCIAS}

1. Barbosa SC, Coledam DHC, Neto AS, Elias RGM, Oliveira AR. Ambiente escolar, comportamento sedentário e atividade física em pré-escolares. Rev Paul Pediatr. 2016;34(3):301-8 doi: 10.1016/j.rppede.2016.02.003

2. Venetsanou F, Kambas A, Gourgoulis V, Yannakoulia M. Physical activity in pre-school children: trends over time and associations with body mass index and screen time. Ann Hum Biol. 2019;46(5):393-9. doi: 10.1080/03014460.2019.1659414

3. Carson V, Langlois K, Colley R. Associations between parent and child sedentary behaviour and physical activity in early childhood. Health Rep. 2020;31(2):3-10. doi: 10.25318/82-003-x202000200001-eng

4. Carson V, Lee EY, Hesketh KD, Hunter S, Kuzik N, Predy M, et al. Physical activity and sedentary behavior across three timepoints and associations with social skills in early childhood. BMC Public Health. 2019;19(1):27. doi: 10.1186/s12889-018-6381-x

5. Venetsanou F, Emmanouilidou K, Kouli O, Bebetsos E, Comoutos $\mathrm{N}$, Kambas A. Physical activity and sedentary behaviors of young children: trends from 2009 to 2018. Int J Environ Res Public Health. 2020;17(5):1645. doi: 10.3390/ijerph17051645

6. Hidding LM, Chinapaw MJ, van Poppel MN, Mokkink LB, Altenburg TM. An updated systematic review of childhood physical activity questionnaires. Sports Med. 2018;48(12):2797842. doi: 10.1007/s40279-018-0987-0

7. Dwyer GM, Hardy LL, Peat JK, Baur LA. The validity and reliability of a home environment preschool-age physical activity questionnaire (Pre-PAQ). Int J Behav Nutr Phys Act. 2011;8(86). doi: 10.1186/1479-5868-8-86

8. Oliveira NKR, Lima RA, Mélo EN, Santos CM, Barros SSH, de Barros MVG. Reprodutibilidade de questionário para medida da atividade física e comportamento sedentário em crianças pré-escolares. Rev Bras Ativ Fis Saude. 2011;16(3):228-33. doi: 10.12820/rbafs.v.16n3p228-233

9. Helmerhorst HJ, Brage S, Warren J, Besson H, Ekelund U. A systematic review of reliability and objective criterion-related 
validity of physical activity questionnaires. Int J Behav Nutr Phys Act. 2012;9:103. doi: 10.1186/1479-5868-9-103

10. Coster WJ, Mancini MC. Recommendations for translation and cross-cultural adaptation of instruments for occupational therapy research and practice. Rev Ter Ocup. 2015;26(1):50-7. doi: 10.11606/issn.2238-6149.v26i1p50-57

11. Beaton DE, Bombardier C, Guillemin F, Ferraz MB. Guidelines for the process of cross-cultural adaptation of self-report measures. Spine. 2000;25(24):3186-91. doi: 10.1097/00007632-200012150-00014

12. Castro Magalhães L. Reminiscências. Cad Ter Ocup. 2012;20(1):143-54. doi: 10.4322/cto.2012.015

13. Braccialli LM, Almeida VS, Sankako AN, Silva MZ, Braccialli AC, Carvalho SM, et al. Translation and validation of the Brazilian version of the Cerebral Palsy Quality of Life Questionnaire for
Children - child report. J Pediatr. 2016;92(2):143-8. doi: 10.1016/j. jped.2015.05.005

14. Guedes DP, Guedes JERP. Medida da atividade física em jovens brasileiros: reprodutibilidade e validade do PAQ-C e do PAQ-A. Rev Bras Med Esporte. 2015;21(6):425-32. doi: 10.1590/1517-869220152106147594

15. Pernambuco L, Espelt A, Magalhães Junior HV, Lima KC. Recomendações para elaboração, tradução, adaptação transcultural e processo de validação de testes em fonoaudiologia. CoDAS. 2017;29(3):e20160217. doi: 10.1590/2317-1782/20172016217

16. Orben A. Teenagers, screens and social media: a narrative review of reviews and key studies. Soc Psychiatry Psychiatr Epidemiol. 2020;55(4):407-14. doi: 10.1007/s00127-019-01825-4 